\title{
CAPACIDADE FUNCIONAL EM INDIVÍDUOS COM DOENÇA PULMONAR OBSTRUTIVA CRÔNICA EM UMA CIDADE DO ALTO PARANAÍBA-MG
}

DOI: 10.22289/2446-922X.V5N2A5

\author{
Heuler dos Reis Rodrigues ${ }^{1}$ \\ Núbia de Fátima Almeida Silva \\ Hugo Christiano Soares Melo \\ Mariane Fernandes Ribeiro \\ Carla Cristina Ferreira de Andrade
}

\section{RESUMO}

A doença pulmonar obstrutiva crônica (DPOC) é uma doença de alta morbimortalidade mundial. È uma doença crônica, progressiva e irreversível caracterizada por limitações ao fluxo aéreo e tosses hipersecretoras. Todos os sinais e sintomas da DPOC acarretam diretamente na capacidade física e funcional dos indivíduos que tem por diagnostico a DPOC. É comum nos indivíduos acometidos pela doença apresentarem perdas e redução progressiva da capacidade física, bem como da capacidade funcional, interferindo diretamente na qualidade de vida dos mesmos. O objetivo deste estudo foi avaliar, por meio do teste de caminhada de seis minutos (TC6min), a capacidade funcional dos portadores da DPOC do Alto Paranaíba. Foram selecionados 08 indivíduos com diagnóstico de DPOC classe GOLD III e GOLDIV de ambos os sexos, com idade média de $67,75 \pm 10,02$ anos, submetidos à avaliação inicial e convidados a participar do TC6min. Durante a realização do teste, foram analisados quanto as variáveis de Pressão Arterial Sistólica (PA), Pressão Arterial Diastólica (PAD), Saturação Periférica de Oxigênio $\left(\mathrm{SpO}^{2}\right)$, Frequência Cardíaca (FC), Distância Percorrida durante o TC6min, Escala Subjetiva de Sensação do Nível de Dispnéia BORG CR 10 modificada. Evidenciou-se correlações positivas estatisticamente significativas $(p<0,05)$ das variáveis $\mathrm{SpO}^{2}, \mathrm{FC}, \mathrm{FR}, \mathrm{PAS}$, BORG CR-10 e distância percorrida em metros em relação ao TC6min. Não havendo diferença estatística, apenas para a variável PAD. Conclui-se que, quanto maior a limitação ao fluxo aéreo, menor a tolerância ao esforço físico pelo paciente. Assim, o TC6min torna-se uma ferramenta importante para avaliar a capacidade funcional do DPOC.

Palavras-chave: Fisioterapia; Pneumopatias; Teste de Caminhada.

\footnotetext{
${ }^{1}$ Endereço eletrônico de contato: heulerrodrigues66@gmail.com

Recebido em 13/11/2019. Aprovado pelo conselho editorial para publicação em 10/12/2019.
}

Rev. Psicol Saúde e Debate. Dez., 2019:5(2):68-81. 


\section{ABSTRACT}

Chronic obstructive pulmonary disease (COPD) is a worldwide disease of morbidity and mortality. It is a chronic, progressive and irreversible disease characterized by airflow changes and hypersecretory releases. All signs and symptoms of COPD directly affect the physical and functional capacity of individuals diagnosed with COPD. It is common in individuals affected by the disease who are shown to progressively reduce and reduce physical capacity and functional capacity, directly interfering with their quality of life. The aim of this study was to evaluate, by means of the six-minute walk test (6MWT), the functional capacity of patients with COPD in Alto Paranaíba. Eight individuals with COPD class GOLD III and GOLDIV of both sexes were selected, with a mean age of $67.75 \pm 10.02$ years, submitted to the initial evaluation and participants of the $6 \mathrm{MWT}$. During the test, we analyzed the variations in systolic blood pressure (BP), diastolic blood pressure (DBP), peripheral oxygen saturation $\left(\mathrm{SpO}^{2}\right)$, heart rate $(\mathrm{HR})$, distance covered during the 6MWT, Subjective Sensing Scale. Dyspnea level BORG CR 10 modified. Statistically significant positive correlations $(p<0.05)$ of the variables SpO ${ }^{2}, \mathrm{HR}, \mathrm{FR}, \mathrm{SBP}, \mathrm{BORG} \mathrm{CR}-10$ and distance covered in meters in relation to the 6MWT were evidenced. There is no statistical difference, only for a PAD variable. In conclusion, the greater the airflow limitation, the lower the patient's tolerance to physical exertion. Thus, the 6MWT becomes an important tool for assessing the functional capacity of COPD. Keywords: Physiotherapy. Functional Training Test. COPD.

Keywords: Physical Therapy Specialty; Lung Diseases; Walk Test.

\section{INTRODUÇÃO}

A doença pulmonar obstrutiva crônica (DPOC) é uma afecção de alta morbimortalidade mundial. Trata-se de uma doença crônica progressiva e irreversível, caracterizada por limitação ao fluxo aéreo e tosses hipersecretoras, que levam a alterações da mecânica respiratória, devido à grande dificuldade de troca gasosa pulmonar acometida pela obstrução brônquica (Barbosa et al., 2017; Miranda et al., 2011).

Segundo Organização Mundial da Saúde (OMS), há no mundo cerca de 65 milhões de portadores da doença, moderada e grave. Ainda de acordo com a OMS, até 2030 a doença será a terceira causa de morte no mundo. O Sistema Único de Saúde (SUS) no Brasil prediz que, entre 1996 á 2013, houve mais de 600 mil mortes oriundas da doença e o crescimento de 12\% desse número entre 2005 e 2010, em que representaram 40 mil óbitos por ano, aumentando os custos financeiros ao SUS devido à grande quantidade de internações, sendo considerado um dos maiores problemas a serem enfrentados pela saúde pública mundial (Cruz \& Costa, 2017; Melo et al., 2018; Freitas et al. 2017).

A principal causa da doença é o tabagismo, que pode ser prevenido. Alguns microorganismos inalados para as vias aéreas também são causas recorrentes para predisposição a doença, tais como aspiração de fumaças, gases tóxicos, fogão a lenha, aspiração de silicose, carvão e poeira produzida nas mineradoras e carvoeiras, falta de maturidade pulmonar e o fator genético pela ausência da proteína alfa 1-anti-tripsina uma, uma 
condição hereditária codominante resultante de diferentes mutações no gene serpina1 (Francisco et al., 2006; Cruz \& Costa, 2017).

As manifestações clínicas da DPOC estão ligadas a uma resposta inflamatória pulmonar anormal. Esta inflamação acarreta uma destruição do parênquima pulmonar, tendo como conseqüência a limitação ao fluxo aéreo que ocorre através de dois mecanismos principais: bronquite crônica, caracterizada por uma tosse hipersecretora com sequência a obstrução brônquica e o enfisema pulmonar, caracterizado pela destruição alveolar e espaçamento anormal dos brônquios aéreos distais. É comum entre estes indivíduos a presença de sinais e sintomas como dispnéia, alteração na ventilação/perfusão, hiperinsuflação pulmonar, respiração sibilante, fadiga durante as práticas de atividade física, perda de retração elástica, destruição alveolar (Dourado et al., 2006; Loivos, 2009; Sousa, 2011).

O diagnóstico da doença é realizado pela história clínica, exames de gasometria arterial, radiografia de tórax e tomografia computadorizada; o padrão ouro para diagnosticar a gravidade da doença é a espirometria, que analisa a funcionalidade pulmonar, por meio do volume de ar que o indivíduo consegue expirar dos pulmões após uma inspiração máxima; este método consiste em avaliar o volume expiratório forçado no primeiro segundo (VEF¹) e a capacidade vital forçada (CVF), para serem comparados a valores previstos pela idade, altura, sexo e etnia, determinando as alterações da gravidade e nível de obstrução da via aérea analisados pelo $V F^{1 / C V F}$, após uso de broncodilatador. A classe funcional é dividida em quatro graus de gravidade, segundo Definição proposta pela Global Initiative for Chronic Obstructive Lung Disease (GOLD), onde GOLD I é leve, representando um baixo risco de exacerbação da doença, sem necessidade de hospitalização; GOLD II moderado, considerado de baixo risco, sem a necessidade de hospitalização apresentando maior risco de exacerbação; GOLD III grave, de alto risco com duas exacerbações ou mais ao ano, com necessidade de hospitalização e menos sintomas; GOLD IV muito grave, alto risco com mais de duas internações por ano, apresentando um maior número de sintomas da DPOC (Dave et al., 2019; Tanet al., 2018; Giacomelli et al., 2014; II Consenso Brasileiro sobre Doença Pulmonar Obstrutiva Crônica, 2004)

Com os avanços da tecnologia, os tratamentos relacionados à DPOC estão se tornando cada vez mais eficazes; em especial, os tratamentos medicamentosos com o uso de corticoides inalatórios, broncodilatadores de curta e longa duração. Deve-se tomar cuidados complementares junto ao uso de fármacos, como a cessação do uso de cigarros e medidas preventivas à exposição dos fatores de risco. Pode-se incluir ainda, como forma de tratamento, terapias com o uso de oxigenoterapia e tratamento cirúrgicos como a bulectomia, lobectomia, pneumectomia e transplante pulmonar. Todos os anos, a OMS juntamente com as sociedades American Thoracic Society European Respiratory Society (ATS/ERS), oferecem uma dosagem de alfa-1-antripsina, beneficiando todos os indivíduos diagnosticados com DPOC, Rev. Psicol Saúde e Debate. Dez., 2019:5(2):68-81. 
independentemente se estiverem assintomáticos ou não. Todavia, para um bom tratamento, é fundamental a individualização dos mesmos, levando em consideração à gravidade da doença e objetivos a serem alcançados (Fernandes et al., 2017; Cruz, 2017).

Segundo a ATS/ERS, a reabilitação do DPOC deve ser realizada por uma equipe multidisciplinar composta por médicos, fisioterapeutas, nutricionistas, enfermeiros, psicólogos e o envolvimento familiar. O fisioterapeuta tem por objetivo atuar na prevenção e reabilitação pulmonar, por meio de exercícios físicos e fortalecimentos, minimizando a seriedade dos sintomas da doença. A fisioterapia deve ser incluída logo após a avaliação cinética funcional, com o intuito de avaliar a gravidade da doença e suas implicações de acordo com cada indivíduo. O fisioterapeuta atuará com técnicas e exercícios respiratórios, com a finalidade de proporcionar a higiene brônquica e aprimorar a capacidade funcional, com a intenção de reduzir a fadiga e dispnéia. As condutas fisioterapêuticas envolvem técnicas de desobstrução brônquica, exercícios de membros inferiores (MMII) e membros superiores (MMSS) para ganho de força e melhora do endurance, exercícios respiratórios diafragmáticos, exercícios respiratórios com resistores de carga linear pressórica, air-stacking e Expiratory Positive Airway Pressure (EPAP). Com decorrência da utilização das condutas, obter uma melhor qualidade de vida nesse grupo de indivíduos (Giacomelli et al. 2014; Sousa, 2019; Corrêa et al., 2011).

A dificuldade de exercitar-se é comum em indivíduos acometidos pela doença. Indivíduos com DPOC tendem a apresentar redução progressiva da sua capacidade física e funcional, por inatividade e alterações do metabolismo glicolítico, interferindo na qualidade de vida desses indivíduos devido à dificuldade de troca gasosa e fraqueza muscular (Fernandes, 2009; Ferreira et al., 2018).

Nos portadores da doença, o recondicionamento físico e funcional gera limitações ventilatórias, sendo comum a utilização de testes físicos e funcionais. Dentre estes, surgiu o teste de caminhada de seis minutos (TC6min), um teste de baixo custo e de utilização mundial, com grande associação às atividades de vida diária (AVDs). O TC6min é um teste submáximo bastante tolerado, que avalia a capacidade funcional e cardiovascular de pessoas doentes e saudáveis. Foi utilizado pela primeira vez em 1970 por Mc Gavin, como uma adaptação do teste de doze minutos sugerido por Cooper em 1968, e aplicado na fisioterapia para avaliar a capacidade funcional de exercício. Consiste em que o paciente caminhe em uma superfície plana, sozinho, sendo capaz de escolher a velocidade e o ritmo da sua caminhada. Também podem receber incentivos verbais, havendo assim uma melhora no desempenho em relação à distância percorrida (Ferrera, 2018; Rodrigues \& Assis, 2002).

Essa pesquisa se dá em razão da alta prevalência de DPOC e da incapacidade funcional causada pela doença nos estágios tardios da mesma. Este estudo objetivou-se em avaliar a capacidade funcional dos indivíduos, com DPOC classe GOLD III e GOLD IV.

Rev. Psicol Saúde e Debate. Dez., 2019:5(2):68-81. 


\section{MATERIAIS E MÉTODOS}

A presente pesquisa trata-se de um estudo transversal, prospectivo, que avaliou a capacidade funcional dos portadores da DPOC GOLD III e GOLD IV por meio do TC6min, e foi realizado na clínica escola de fisioterapia de uma instituição de ensino superior de uma cidade do Alto Paranaíba-MG.

A pesquisa foi aprovada pelo Comitê de Ética e Pesquisa com seres humanos, com o número do parecer 3.287.183. Todos os integrantes do estudo foram devidamente informados sobre a pesquisa e assinaram o Termo de Consentimento Livre e Esclarecido (TCLE), onde estão descritas todas as informações sobre a mesma, conforme a resolução CNS n.466 de 12 de dezembro de 2012.

Os critérios de inclusão foram: indivíduos com DPOC GOLD III e GOLD IV, de ambos os sexos e faixa etária de idade média de 67,75 $\pm 10,02$ anos; não apresentarem nenhum tipo de seqüela neurológica nem déficits de MMII e demências; apresentarem estabilidade clinica sem exacerbação da DPOC nas últimas seis semanas; ausência de comprometimentos neuromusculares que limitassem a realização do TC6min. Para os critérios de exclusão foram observados todos os participantes que não se enquadraram nos parâmetros de inclusão e não estarem de acordo com o TCLE.

A amostra constituiu-se de 08 indivíduos com diagnóstico da DPOC divididos em GOLD III e GOLD IV. Sendo três GIII e cinco da GIV, cinco mulheres e três homens. Todos os indivíduos foram submetidos à avaliação inicial em anexo $(A)$ e, em seguida, convidados a participar do TC6min, observando as subsequentes variáveis antes e depois do teste: Pressão Arterial Sistólica (PAS), Pressão Arterial Diastólica (PAD), Frequência Cardíaca (FC), Saturação Periférica de Oxigênio (SPO²), Frequência Respiratória (FR), Escala de BORG CR10 para o apontamento de dispnéia em anexo $(B)$ e Distância Percorrida para ser comparada à Distância Predita. Os materiais aplicados para execução da pesquisa foram estetoscópio Premium, esfigmomanômetro Premium, oxímetro de pulso portátil modelo G-TECH de alta precisão e cronômetro (Ferrera et al., 2018; Rodrigues \& Asis, 2002; Silva et al., 2012).

Antes da realização do teste, ainda na coleta de dados, calculou-se a frequência cardíaca máxima prevista para cada idade, utilizando-se o divisor em porcentagem de $85 \%$, utilizada como critério de segurança para realização do teste (Britto, 2017).

O TC6min foi realizado no corredor de 20 metros por 2 metros de largura em linha reta na Clínica Escola da Faculdade Patos de Minas. Os integrantes foram orientados a caminhar durante 6 minutos de forma rápida, estimulados por expressões padronizadas pela ATS, tais como: "você consegue!"; "você pode mais!"; "vamos mais rápido!" e acompanhados durante todo o percurso. Todos foram monitorados quanto a $\mathrm{SpO}^{2}$ e $\mathrm{FC}$ através de um oxímetro de pulso. Os

Rev. Psicol Saúde e Debate. Dez., 2019:5(2):68-81. 
integrantes que apresentaram queda da $\mathrm{SpO}^{2}$ abaixo de $80 \%$, foram ofertados oxigênio para alcançar um valor de 92\%. Para os indivíduos que precisaram desfadigar ou reduzira velocidade da caminhada, o teste foi interrompido e o cronômetro continuou ligado até o sexto minuto; não obstante, o indivíduo foi encorajado a retomar o teste o mais rápido possível, registrando-se antes e depois do teste a FR, PAS, PAD, Escala de BORG CR10 e a Distância Percorrida em metros para serem comparados de acordo com valores previstos por Enright e Sherril (Da Costa Teixeira et al., 2018; Resende et al., 2017).

Homens DPprev $=(7,57 \times$ altura $\mathrm{cm})-(5,02 \times$ idade $)-(1,76 \times$ peso $\mathrm{kg})-309$ metros.

Mulheres DPprev $=(2,11 \times$ altura $\mathrm{cm})-(2,29 \times$ idade $)-(5,78 \times$ peso $\mathrm{kg})-667$ metros.

DPprev = distância percorrida prevista no TC6min.

Para análise dos resultados utilizou-se cálculo do teste $t$ de Student pareado, com o software Graphpad Prism.

\section{RESULTADOS E DISCUSSÃO}

O presente estudo teve por finalidade avaliar a capacidade funcional dos indivíduos da DPOC GOLD III e GOLD IV, utilizando-se o TC6min como ferramenta para coleta de dados. Acredita-se que, o pequeno número da amostra, se dê em razão da inconfiabilidade médica e dos profissionais da saúde, em realizar o encaminhamento desses pacientes para coleta de dados, muitas vezes evidenciado por falta de interesse e medo de indicação pela gravidade da doença.

Constatou-se diferença estatística de $\mathrm{SpO}^{2}$, quando comparados $\mathrm{SpO}^{2}$ inicial e $\mathrm{SpO}^{2}$ final, indicando média inicial $(94,6 \pm 2,9)$ e média final $(91,0 \pm 4,3)$. A análise foi feita pelo teste $t$ de student pareado, indicando $(p<0,0288)$ com diferença estatística significativa $(p<0,05)$ ao comparar $\mathrm{Spo}^{2}$ inicial e $\mathrm{SpO}^{2}$ final, como demostrado na figura 1.

Rev. Psicol Saúde e Debate. Dez., 2019:5(2):68-81. 


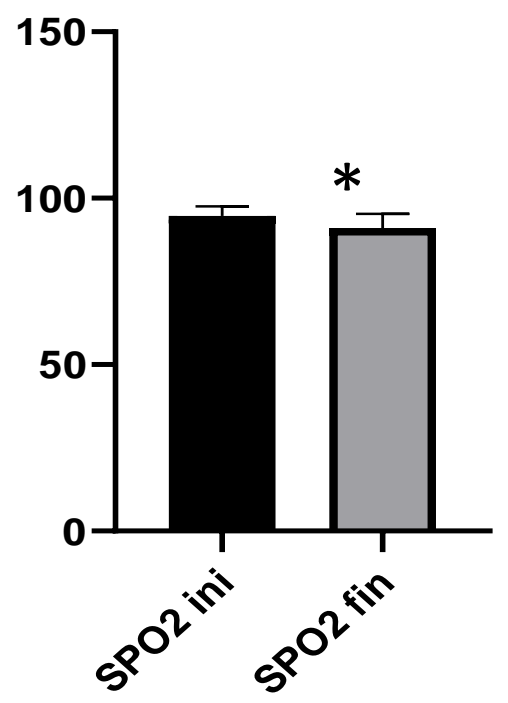

Figura 1. Saturação periférica de Oxigênio $\left(\mathrm{SpO}^{2}\right)$ antes e depois do TC6min. * indica uma diferença estatisticamente significante $(p \leq 0,05)$.

Em achados de um estudo, percebeu-se que o esforço produzido durante o TC6min, possibilitou o aumento significativo da $\mathrm{FC}$, fator que induziu a queda de $\mathrm{SpO}^{2}$ dos indivíduos, sendo justificado pelas alterações ventilação/perfusão (Morales-Blanhir, 2011).

Demonstrou-se diferença estatística significativa da FC antes do TC6min, média inicial $(74,5 \pm 20,1)$ e média final $(104,9 \pm 26,5)$ com valor de $(p<0,0167)$, ao comparar FC inicial e FC final, demostrado na figura 2 .

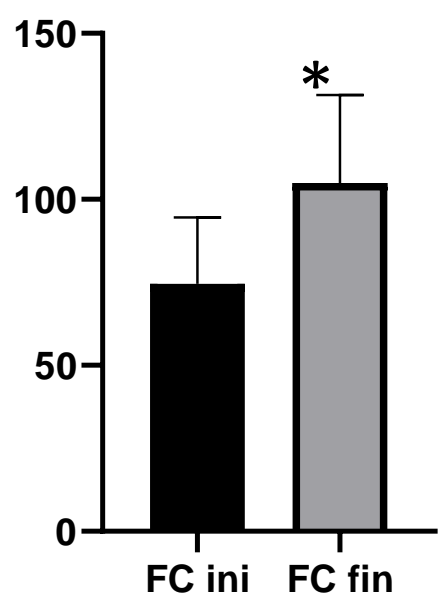

Figura 2. Frequência Cardíaca (FC) antes e depois do TC6min. * indica uma diferença estatisticamente significante $(p \leq 0,05)$. 
Verificou-se ainda que, aproximadamente $60 \%$ dos indivíduos, ultrapassaram a frequência cardíaca máxima ao final do teste e, quando comparado a FC antes e após o TC6min, observou-se um aumento significativo da FC após a realização do TC6mim, fator que pode ser atribuído ao grande esforço durante o teste, levando a alterações hemodinâmicas incluindo a FC por aumento do débito cardíaco (Morales-Blanhir, 2011).

A figura 3, ao ser analisada identificou-se diferença estatística significativa de FR para antes e depois do TC6min, com média inicial $(22,00 \pm 5,9)$ e média final $(31,6 \pm 7,5)$, indicando valor de $(p<0,0033)$ ao comparar FR inicial e FR final.

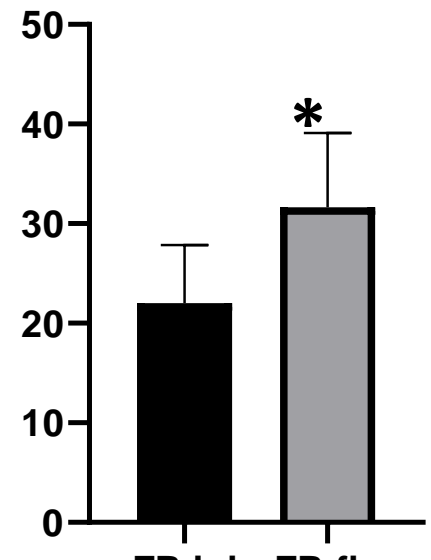

FR ini FR fin

Figura 3. Frequência Respiratória (FR) antes e depois do TC6mim. * indica uma diferença estatisticamente significante $(p \leq 0,05)$.

Verificou-se, em um estudo retrospectivo, onde foram realizados dois TC6min em dias alternados, que não houve diferença estatística significativa na FR em ambos os testes. Pode ser pela razão de que o estudo analisado não delimitou quais as classes funcionais participariam do estudo, divergindo-se com os resultados aqui encontrados; acredita-se que, por se tratarem de indivíduos classe GOLD III e GOLD IV, as limitações e respostas hemodinâmicas encontradas nesses grupos sejam maiores por se tratar de fases mais avançadas da doença (Silva, 2019).

Foi observado na figura 4, diferença estatística significativa para BORG CR-10 entre o período de antes e depois do TC6min, com valor de média inicial $(2,9 \pm 2,0)$ e média final $(6,4 \pm$ $2,2)$ com valor de ( $p<0,0109)$, ao comparar BORG CR-10 inicial e BORG CR-10 final. 


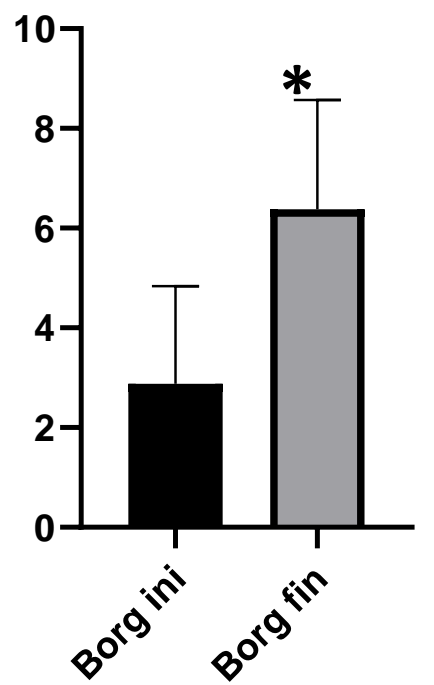

Figura 4. BORG CR-10 antes e depois do TC6min. * indica uma diferença estatisticamente significante $(p \leq 0,05)$.

A falta de ar nos indivíduos com DPOC denominada dispnéia, é um sintoma caracterizado como uma sensação de respiração desagradável. Não há, atualmente, exames laboratoriais ou testes funcionais que possam quantificar o nível de dispnéia que contemplem todos os aspectos desse sintoma; todavia, existem escalas unidimensionais que quantificam o nível da sensação de dispnéia. Em um estudo com a finalidade de verificar a tolerância ao exercício de indivíduos com DPOC, verificou-se que a escala de BORG CR 10, é útil para mensurar a sensação do nível de dispnéia, após confrontarem a tabela com mais supostos da literatura frente ao TC6min (Rodrigues, 2004, \& Camargo, 2010).

Ao analisar a figura 5, constatou-se diferença estatística significativa para PAS entre o período de antes e depois do TC6min, com valor de média inicial $(126,3 \pm 19,2)$ e média final $(152,4 \pm 41,3$ ), indicando ( $p<0,0286$ ), ao comparar PAS inicial e PAS final. Por outro lado, observou-se que não houve diferença estatística significativa quando comparados PAD inicial, média $(81,3 \pm 8,3)$ e final média $(90,6 \pm 13,2)$ indicando $(P<0,0,0541)$, ao comparar PAD inicial e PAD final como demostrado na Figura 5. 
PA sistolica

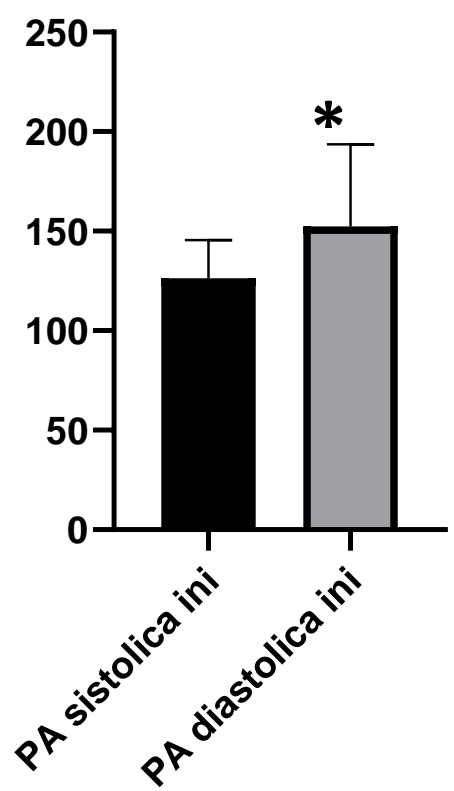

PA diastolica

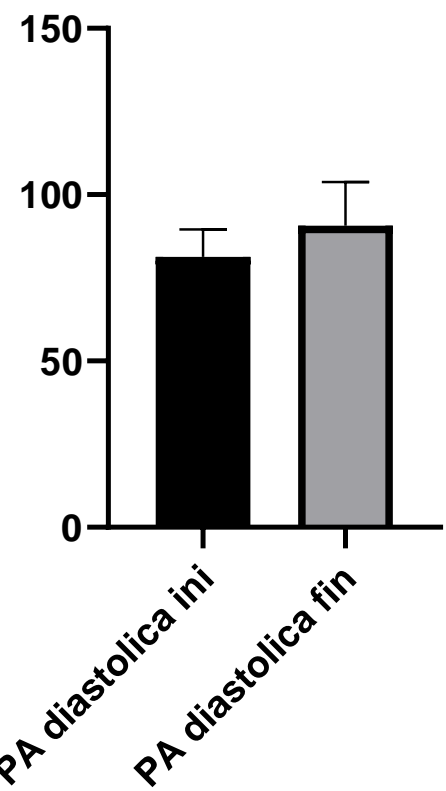

Figura 5. PAS inicial, PAS final, PAD inicial e PAD final. Para ambas as variáveis analisadas antes e depois do TC6min, em mm de Hg. ${ }^{*}$ indica uma diferença estatisticamente significante $(p \leq 0,05)$.

O esforço gerado durante o TC6min por indivíduos DPOC, aponta aumento significativo da PA sistólica, não evidenciando mudanças significativas para PA diastólica após o TC6min. A inatividade dos indivíduos com DPOC gera uma intolerância a prática de atividades físicas, causando um déficit no sistema cardiovascular. Como no caso deste, um estudo demonstrou que houve um aumento significativo da PAS, em indivíduos DPOC submetidos a esforços físicos utilizando o TC6min, para suprir a demanda metabólica durante a pratica de atividade física, não estando relacionado com a obstrução brônquica apresentada por esses indivíduos, mas pela inatividade ou problemas cardiovasculares (Camargo, 2010, \& Schettino, 2013).

A figura 6 quando comparada a Distância Prevista $\times$ Distância Percorrida mostrou-se diferença significativa para GIII média ( $241 \pm 88 \mathrm{~m}$ vs $587 \pm 186 \mathrm{~m})$ e GIV média $(240 \pm 219 \mathrm{~m}$ vs605 $\pm 208 \mathrm{~m}$ ), sendo a percorrida menor que a prevista para ambos. Na análise do grupo, quanto à distância percorrida e a prevista, verificou-se diferença significativa $(p \leq 0,05)$, sendo que GIV apresentaram valores maiores de distância prevista que o GIII. 


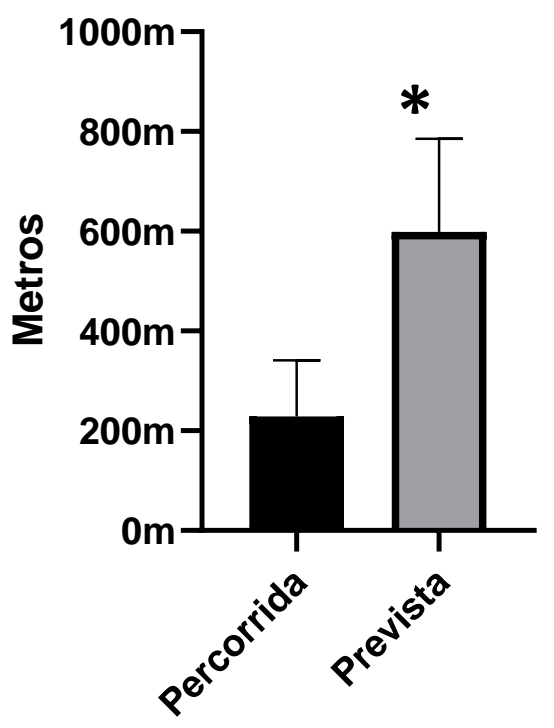

Figura 6. Distância Prevista antes do TC6min e Distância Percorrida depois do TC6min. * indica uma diferença estatisticamente significante $(p \leq 0,05)$.

O TC6min até hoje é o mais importante e principal teste para avaliar a capacidade física de indivíduos com limitações funcionais, bem como a gravidade da incapacidade funcional. Os mesmos autores declaram que o TC6min é o teste mais aplicado para avaliar a capacidade funcional dos indivíduos com DPOC e afirmam ainda não haver diferença significativa, ao comparara distância percorrida da distância prevista para tais indivíduos; porém, notaram diferença significativa ao comparar a distância percorrida com distância prevista, para indivíduos DPOC com graus de obstrução de moderado a grave. Confirma-se o encontrado neste estudo, quando comparado a distância percorrida da prevista para os indivíduos de maior grau de obstrução sendo eles DPOC GOLD III e GOLD IV (Queiroz, 2015).

\section{CONSIDERAÇÕES FINAIS}

Conclui-se que, quanto maior a gravidade da doença, os indivíduos com DPOC apresentaram maior limitação ao fluxo aéreo e menor tolerância ao esforço físico; objetivando a necessidade da avaliação da capacidade funcional, a fim de verificar prognósticos e avaliação terapêutica adequada para esta determinada classe. Assim o TC6min representa uma ferramenta importante para avaliação da capacidade funcional do DPOC. 


\section{REFERÊNCIAS}

Barbosa, A. T. F., Carneiro, J. A., Ramos, G. C. F., Leite, M. T., \& Caldeira, A. P (2017). Fatores associados à doença pulmonar obstrutiva crônica em idosos. Ciência e Saúde Coletiva, 22(1), 63-73. doi: 10.1590/1413-81232017221.13042016.

Britto, R., \& de Sousa, L (2017). TESTE DE CAMINHADA DE SEIS MINUTOS UMA NORMATIZAÇÃO BRASILEIRA. Fisioterapia em Movimento, 19(4). Retirado de https://periodicos.pucpr.br/index.php/fisio/article/view/18789

Camargo, L. A. C. R., \& Pereira, C. A. C (2010). Dispneia em DPOC: além da escala modified Medical Research Council. Jornal Brasileiro de Pneumologia, 36(5), 571-578. doi: 10.1590/S1806-37132010000500008.

Corrêa, K. S., Karloh, M., Martins, L. Q., Santos, K., \& Mayer, A. F (2011). O teste de AVD-Glittre é capaz de diferenciar a capacidade funcional de indivíduos com DPOC da de saudáveis? Brazilian Journal of Physical Therapy, 15(6), 467-473. Epub 21 de novembro de 2011. doi: 10.1590/S1413-35552011005000034.

Cruz, T.F., \& Costa, C. H (2017). Deficiência de alfa-1antitripsina: uma condição subdiagnosticada. Pulmão RJ, 26(1), 29-32. Retirado de http://www.sopterj.com.br/wpcontent/themes/_sopterj_redesign_2017/_revista/2017/n_01/07-artigo.pdf.

Da Costa Teixeira, P., Pereira, L., \& Vieira, K (2018). Teste de caminhada de seis minutos e suas aplicabilidades: uma revisão sistemática da literatura. Revista Valore, 3(2), 646-662. doi: 10.22408/reva32201887646-662.

Dave, S., Alvar, A., Antonio, A., Peter, J. B., Jean, B., Bartolome, R. C., Gerard, J. C., Peter, F., David, M. G., Halpin, M. H., Victorina, L. V., Fernando, M., Maria, M. O., Alberto Papi, I. D. P., Nicolas, R., Donald, D. S., Robert, S., Jorgen, V., Jadwiga, A. W, \& ClausVogelmeier (2019). Global Strategy for the Diagnosis, Management, and Prevention of Chronic Obstructive Lung Disease: the GOLD science committee report 2019. European Respiratory Journal, 53: 1900164; doi: 10.1183/13993003.00164-2019.

Dourado, V. Z., Tanni, S. E., Vale, S. A., Faganello, M. M., Sanchez, F. F., \& Godoy, I (2006). Manifestações sistêmicas na doença pulmonar obstrutiva crônica. Jornal Brasileiro de Pneumologia, 32(2), 161-171. doi: 10.1590/S1806-37132006000200012.

Fernandes, A. B. S (2009). Reabilitação respiratória em DPOC - a importância da abordagem fisioterapêutica. Pulmão RJ - Atualizações Temáticas, 1(1), 71-78. Retirado de http://www.sopterj.com.br/wpcontent/themes/_sopterj_redesign_2017/_revista/atualizacao_ tematica/11.pdf.

Fernandes, F. L. A., Cukier, A., Camelier, A. A., Fritscher, C. C., Costa, C. H., Pereira, E. D. B., Cançado, J. E. D., Romaldini, J. G., Chatkin, J. M., Jardim, J. R., Rabahi, M. F., Nuci, M. C. N. M., Sales, M. P. U., Castellano, M. V. C. O., Aidé, M. A., Teixeira, P. J. z., Maciel, R., Correia, R. A., Stirbulov, R., Athanazio, R. A., Russo, R., Minamoto, S. T., Lundgren, F. L. C (2017). Recomendações para o tratamento farmacológico da DPOC: perguntas e respostas. J Bras Pneumol, 43(4), 290-301. doi: 10.1590/S1806-37562017000000153.

Ferreira, D., Schneiders, P., Mansour, K., Martins, D., Back, G., Martin, E., Goulart, C., Carvalho, L., Trimer, R., \& Silva, A (2018). Efeitos da Pressão Positiva Expiratória sobre a capacidade de exercício em sujeitos acometidos por Doença Pulmonar Obstrutiva Crônica. Revista Jovens Pesquisadores, 8(2), 20-30. doi: 10.17058/rjp.v8i2.11827.

Rev. Psicol Saúde e Debate. Dez., 2019:5(2):68-81. 
Francisco, P. M. S. B., Donalisio, M. R., Barros, M. B. A., César, C. L. G., Carandina, L., \& Goldbaum, M (2006). Fatores associados à doença pulmonar em idosos. Revista de Saúde Pública, 40(3), 428-435. doi: 10.1590/S0034-89102006000300010.

Freitas, A., Chaves, J., Krummenauer, M., Tomilin, B., Ourique, F., Fuhr, L., Porciúncula, A., Frey, A., Rodrigues, M., Pilletti, K., Fernandes, R., \& Rodrigues, M (2017). Study COPD Diagnosis Prevalence in Patient Admitted with Ischemic Heart Disease in University, 7(1), 14-19. doi: 10.17058/reci.v7i1.7348.

Giacomelli, I. I., Steidle, L.J.M., Moreira, F.F., Meyer, I.V., Souza, R.G., \& Pincelli, M.P (2014). Pacientes portadores de DPOC hospitalizados: análise do tratamento prévio. J. bras. pneumol, 40(3), 229-237. doi: 10.1590/S1806-37132014000300005.

II Consenso Brasileiro sobre Doença Pulmonar Obstrutiva Crônica (DPOC) (2004). Caracterização da Doença Pulmonar Obstrutiva Crônica (DPOC) - Definição, Epidemiologia, Diagnóstico e Estadiamento. Sociedade Brasileira de Pneumologia e Tisiologia. J Bras Pneumol:; 30(5). Disponível em: http://www.jornaldepneumologia.com.br/pdf/suple_124_40_dpoc_completo_finalimpresso.p df.

Loivos, L. P (2009). DPOC: definições e conceitos - bases clínicas. Rev. Atualizações Temáticas. RJ, 1:34-37. Retirado de http://www.sopterj.com.br/wpcontent/themes/_sopterj_redesign_2017/_revista/atualizacao_tematica/04.pdf.

Melo, T. G., Santoni, N. B., Finkelstein, B. J., Veiga, D. L. P., Nascimento, M. H. S. R., \& Arruda, C. A (2018). Índice de hospitalização e custos associados à doença pulmonar obstrutiva crônica (DPOC) entre estados que não padronizaram versus que não padronizaram o tiotrópio: Dados do Mundo Real. J Bras Econ Saúde, 10(1), 29-35. doi: 10.21115/JBES.v10.n1.p29-35.

Miranda, E. F., Malaguti, C., \& Corso, S. D (2011). Disfunção muscular periférica em DPOC: membros inferiores versus membros superiores. Jornal Brasileiro de Pneumologia, 37(3), 380-388. doi: 10.1590/S1806-37132011000300016.

Morales-Blanhir, J. E., Vidal, C. D. P., Romero, M. J. R., Castro, M. M. G., Villegas, A. L., \& Zamboni, M (2011). Teste de caminhada de seis minutos: uma ferramenta valiosa na avaliação do comprometimento pulmonar. Jornal Brasileiro de Pneumologia, 37(1), 110-117. doi: 10.1590/S1806-37132011000100016.

Queiroz, J. R., Sérvio, T. C., \&Barbieri, L. G (2015). Relação entre o teste da caminhada de 6 minutos e a percepção subjetiva de esforço em indivíduos com DPOC: uma revisão de literatura. Revista Digital. Buenos Aires. [periódico na internet]. 2015 [acesso em 10 Nov 2019]; 20(206). Retirado de https://www.efdeportes.com/efd206 /percepcao-subjetiva-deesforco-com-dpoc.htm.

Rezende, R. R., Nogueira, O. A., Dal Corso, S., \& Malaguti, C (2017). Uma atualização e proposta de padronização do teste de caminhada dos seis minutos. Fisioterapia em Movimento, 22(2). Retirado de https://periodicos.pucpr.br/index.php/fisio/article/view/19427/18769.

Rodrigues, S. L., \& ASSIS VIEGAS, C. A (2002). Estudo de correlação entre provas funcionais respiratórias e o teste de caminhada de seis minutos em pacientes portadores de doença pulmonar obstrutiva crônica. Jornal de Pneumologia, 28(6), 324-328. doi: 10.1590/S010235862002000600005 .

Rodrigues, S. L., Mendes, H. F., \& Viegas, C. A. A (2004). Teste de caminhada de seis minutos: estudo do efeito do aprendizado em portadores de doença pulmonar obstrutiva crônica.

Rev. Psicol Saúde e Debate. Dez., 2019:5(2):68-81. 
Jornal Brasileiro de Pneumologia, 30(2), 121-125. doi: 10.1590/S180637132004000200008.

Schettino, C. D. S., Deus, F. C., Gonçalves, A. A. V., \&Wallace E (2013). Relação entre DPOC e doença cardiovascular. Rev. Pulmão RJ, 22(2):19-23. Retirado de http://www.sopterj.com.br/wpcontent/themes/_sopterj_redesign_2017/_revista/2013/n_02/0 5.pdf.

Silva, C.B., Gonçalves. K. D., Silveira, J. M., Reis, G. R., Ueda, T. K (2012). Comparação da distância percorrida em três modalidades do teste de caminhada de seis minutos com equações preditivas. ASSOBRAFIR. 3(3), 19-29. Retirado de http://www.uel.br/revistas/uel/index.php/rebrafis/article/view/11744.

Silva, J. R. O., Santana, J. R., Silva, C. C., Simões, S., Camelier, A. A., \&Camelier, F. W. R (2019). Cardiovascular adaptation in thesix-minute walktest in COPD patients: transversal study. Rev Pesqui Fisioter, Salvador, 9(1):56-66. doi: 10.17267/2238-2704rpf.v9i1.2224.

Sousa, C. A., César, C. L. G., Barros, M. B. A., Carandina, L., Goldbaum, M., \& Pereira, J. C. R (2011). Doença pulmonar obstrutiva crônica e fatores associados em São Paulo, SP, 20082009. Revista de Saúde Pública, 45(5), 887-896. Epub July 29, 2011. doi: 10.1590/S003489102011005000051.

Souza, A. J. T., \& Schneider, I. F (2019). A importância da atuação fisioterapêutica para manter a qualidade de vida dos pacientes com doença pulmonar obstrutiva crônica - DPOC. Revista Científica da faculdade de educação e meio ambiente, 10(1), 168-177. doi: 10.31072/rcf.v10iedesp.795.

Tan, W. C., Bourbeau, J., Aaron, S. $\quad$ D., Zhou, G., Maltais, F, \&Hernandez, $\quad$ P. et al (2018). Classific ação global iniciativa para doença pulmonar obstrutiva crônica 2017 e declínio da função pulmonar na doença pulmonar obstrutiva crônica. Am J Respir Crit Care Med, 197:670-673. doi: 10.1164 / rccm.201706-1154LE. 